\title{
A Much Sought-After Drug_Propofol Sedation for GI Endoscopy: Always Better but Who Cares?
}

\author{
Lorella Fanti · Marco Gemma · Massimo Agostoni • \\ Pier Alberto Testoni
}

Received: 29 May 2012/ Accepted: 7 June 2012/Published online: 29 June 2012

(C) Springer Science+Business Media, LLC 2012

Several hypnotic and analgesic strategies have been devised for patients undergoing gastrointestinal (GI) endoscopy, ranging in a continuum from mild conscious sedation to general anesthesia.

Endoscopic retrograde cholangiopancretography (ERCP) and endoscopic ultrasound (EUS) are particularly unpleasant diagnostic procedures for patients and more time consuming than any other endoscopic procedure. Moreover, ERCP can be technically difficult and requires complete patient cooperation in order to be safely and successfully completed. Patients frequently are sedated for ERCP or EUS with intravenous bolus doses of benzodiazepines in combination with opioids, which are meant to improve the quality of sedation by providing analgesia. Deep sedation is sometimes required.

The introduction of propofol into clinical practice significantly changed the attitudes of both physicians and patients towards sedation, with the result that propofol sedation for GI endoscopy has become common practice worldwide. In recent years, propofol has been safely and effectively used in advanced interventional endoscopic procedures, such as ERCP and EUS, even for high-risk patients [1]. Propofol is a hypnotic drug with rapid onset and offset of action. Used as a single agent, it is commonly titrated to attain deep sedation, whereas balanced propofol sedation (BPS), in which propofol use is associated with

\footnotetext{
L. Fanti $(\bowtie) \cdot$ P. A. Testoni

Division of Gastroenterology and Gastrointestinal Endoscopy, Scientific Institute San Raffaele Hospital, Vita-Salute San Raffaele University, Via Olgettina 60, 20132 Milan, Italy e-mail: fanti.lorella@hsr.it

M. Gemma · M. Agostoni

Department of Anesthesiology, Scientific Institute San Raffaele Hospital, Vita-Salute San Raffaele University, Milan, Italy
}

small doses of benzodiazepines and/or opioids, can be successfully titrated to attain moderate sedation.

Several studies have shown that, when compared with conventional sedation, BPS with propofol, midazolam, and/or meperidine, yields higher physician and patient satisfaction, better patient cooperation, and similar complication rates in patients undergoing therapeutic endoscopic procedures. The total propofol dose may be expected to be reduced by $50 \%$ with BPS in comparison with propofol alone sedation.

A critical advantage in dealing with BPS for moderate sedation is that it can be partially reverted, since antagonists are available against both opioids and benzodiazepines.

In the current issue of Digestive Diseases and Sciences, Tae Hoon Lee et al. [2] present the results of a randomized prospective study comparing BPS (propofol, midazolam, and fentanyl) with propofol alone during moderate sedation for ERCP and EUS. The authors demonstrate that propofol alone provides a shorter recovery time than BPS and is comparably effective and safe. This paper is the natural evolution of a previous study of the same authors in which they demonstrated a higher safety and efficacy of BPS in comparison with conventional sedation [3]. In this respect, Tae Hoon Lee et al. positively contribute to the knowledge of sedation strategies for endoscopy.

However, in another respect, they quietly and almost inadvertently hit a raw nerve in the ongoing debate about who can/should provide propofol sedation. In both of their studies, sedation was provided by non-anesthesiologists, further adding to the body of literature indicating that the administration of propofol by non-anesthesiologists may be safe and effective for selected patients undergoing advanced endoscopic procedures.

The subject of non-anesthesiologist administration of propofol (NAAP), particularly for endoscopy, has 
generated a mass of studies in which propofol has been administered by either physicians or nurses who have received appropriate training. The worldwide experience on NAAP now exceeds 640,000 patients [4], and collected data suggest that NAAP can be safe and effective if appropriate patient selection is applied and the involved non-anesthesiologists have acquired adequate skill and knowledge through dedicated theoretical and practical training programs. Moreover, although current propofol administration protocols are safe and effective for the majority of patients, much interest has focused on techniques that enhance patient satisfaction, safety, and recovery: a very desirable and particularly useful topic in the NAAP setting.

Dumonceau, in a recent editorial, cited propofol infusion platforms as a promising option for NAAP since they allow a better drug delivery control and could contribute to a further lowering of costs and easing of medico-legal concerns [5]. Established trends in sedation include enhanced mechanisms for propofol delivery, such as target-controlled infusion (TCI) and patient-controlled sedation or analgesia (PCS or PCA). The American Society of Anesthesiologists (ASA) guidelines recommend accurate titration of sedative/analgesic drugs to improve patient's comfort and safety, with the specific recommendation to limit the risk of oversedation. Among the different methods now available for propofol administration, TCI is undoubtedly one of the most sophisticated and propofol TCI has proved to be an excellent and safe procedure during sedation for endoscopic procedures [1]. Technological developments are rapidly advancing with the advent of computer-assisted personalized propofol administration systems (such as the SEDASYS ${ }^{\mathrm{TM}}$ (Ethicon Endo-Surgery, Cincinnati, $\mathrm{OH}$ ), which effectively assist physician-nurse teams to maintain minimal-to-moderate sedation levels throughout GI endoscopy, thereby limiting the risk of inadvertent deep sedation and its mostly feared consequence, cardio-respiratory failure [6].

Such emerging technologies will develop hand-in hand with the proper training to lay the foundations for NAAP teams qualified for GI endoscopy. However, such steps are often easier said than done, and the NAAP topic has recently been the focus of fierce debates in the scientific arena.

The virtues of propofol sedation have lured endoscopists to autonomously administer this hypnotic drug-even though manufacturers' recommendations limit its use to anesthesiologists. The great number of publications generated on propofol sedation for endoscopy has been partially triggered by the desire to support the NAAP, and several concerns have been raised during the last decade which are as yet unresolved. In the USA, The American Association for the Study of Liver Disease, the American
College of Gastroenterology, the American Gastroenterological Association, and the American Society for Gastrointestinal Endoscopy together have established a pro-NAAP front; as such, they are in strong disagreement with the ASA, which opposes NAAP. The governing boards of these four gastroenterology societies developed a joint statement to endorse an evidence-based assessment on the safety, efficacy, and cost-effectiveness of NAAP for GI endoscopy. The final document was approved by the respectively four governing boards and was published jointly in 2009 in Gastrointestinal Endoscopy, American Journal of Gastroenterology, and Journal of Gastroenterology and Hepatology [7].

The resulting guidelines state that NAAP is more costeffective than standard sedation with benzodiazepine and opioids and is comparable in terms of efficacy and safety profiles. The sedation task force, chaired by L.B. Cohen, plainly states that, even for ERCP and EUS, NAAP is more cost-effective than standard sedation and that anesthesiologist-administered sedation for healthy, low-risk patients undergoing routine gastrointestinal endoscopy results in higher costs and has no proven benefits in terms of patient safety or procedural efficacy.

In Europe this topic has recently become even more controversial. The December 2010 issue of The European Journal of Anaesthesiology, together with the European Society of Anaesthesiology (ESA), the European Society of Gastrointestinal Endoscopy (ESGE), and the European Society of Gastroenterology and Endoscopy Nurses and Associates (ESGENA), endorsed a joint evidence- and consensus-based set of guidelines on NAAP for gastrointestinal endoscopy. These guidelines were concurrently published in Endoscopy [8]. Publication of these guidelines has resulted in a bitter debate in Europe. Due to vehement criticism from 21 European national societies of anesthesia [9], the European Society of Anesthesiology had to retract its recently issued endorsement that had opened the door to NAAP. The retraction appeared in a letter by P. Pelosi, written on behalf of the Board of the European Society of Anaesthesiology, in the April 2012 issue of the European Journal of Anaesthesiology [10]. The same issue of the European Journal of Anaesthesiology correctly hosted a reply to the retraction letter written by J.M. Dumonceau on behalf of the NAAP task force [11]. Dumonceau took a tough position against the retraction, expressing "astonishment" about the case and stating that, in the absence of any new scientific evidence contradicting the published guidelines, European gastroenterology societies continued to endorse them.

This conflict is deep-rooted has been around a very long time; recent events have simply exacerbated the debate. In his editorial [5], J.M. Dumonceau also voiced a diffuse opinion that openly indicates that the major hurdles for 
NAAP implementation are the enormous financial incentives derived from anesthesiologist-assisted endoscopic procedures and the institutional control maintained by anesthesiologists over sedation policies. An expression of these hurdles would be the immutable black-box warning about propofol administration: current propofol package labeling still states that only anesthesiologists should administer the drug, which is in total disregard of all successive evidence on the NAAP safety profile.

In a recent thorough cost-effectiveness analysis comparing endoscopist- versus anesthesiologist-directed propofol administration, Hassan et al. [12] provide evidence showing that the potential benefit of NAAP implementation for screening colonoscopy would be huge in both the American and European setting. The conclusion of their report is particularly relevant: "When the saving of economic and medical resources is borne in mind, any resistance that is not evidence-based should yield to a new, constructive, and open-minded perspective."

It is inconvenient that specialists of different disciplines quarrel about clinical practices that would require effective cooperation to be acceptably fulfilled. What should be taken for granted is that in an open scientific debate between opposite fronts, only evidence should be provided by both parts to support their respective positions.

\section{References}

1. Agostoni M, Fanti L, Gemma M, et al. Adverse events during monitored anesthesia care for GI endoscopy: an 8-year experience. Gastrointest Endosc. 2011;74:266-275.

2. Lee TH, Lee CK, Park SH et al. Balanced propofol sedation versus propofol monosedation in therapeutic pancreaticobiliary endoscopic procedures. Dig Dis Sci. 2012. (Epub ahead of print). doi:10.1007/s10620-012-2234-0.
3. Lee CK, Lee SH, Chung IK, et al. Balanced propofol sedation for therapeutic GI endoscopic procedures; a prospective, randomized study. Gastrointest Endosc. 2011;73:206-214.

4. Rex D, Deenadalayu V, Eid E, et al. Endoscopist-directed administration of propofol: a worldwide safety experience. Gastroenterology. 2009;137:1229-1237.

5. Dumonceau JM. Nonanesthesiologist administration of propofol: it's all about money. Endoscopy. 2012;44:453-455.

6. Pambianco DJ, Whitten CJ, Moerman A, et al. An assessment of computer-assisted personalized sedation: a sedation delivery system to administer propofol for gastrointestinal endoscopy. Gastrointest Endosc. 2008;68:542-547.

7. Vargo JJ, Cohen LB, Rex DK, et al. Position statement: nonanesthesiologists administration of propofol for GI endoscopy. Gastroenterology. 2009;137:2161-2167.

8. Dumonceau JM, Riphaus A, Aparicio JR, et al. European Society of Gastrointestinal Endoscopy, European Society of Gastroenterology and Endoscopy Nurses and Associates and the European Society of Anesthesiology Guideline: non-anaesthesiologist administration of propofol for GI endoscopy. Eur J Anaesthesiol. 2012;27:1016-1030.

9. Perel A. Non-anaesthesiologists should not be allowed to administer propofol for procedural sedation: a consensus statement of 21 European National Societies of Anaesthesia. Eur J Anaesthesiol. 2011;28:580-584.

10. Pelosi P. Retraction of endorsement: European Society of Gastrointestinal Endoscopy, European Society of Gastroenterology and Endoscopy Nurses and Associates and the European Society of Anaesthesiology guideline-non-anaesthesiologist administration of propofol for gastrointestinal endoscopy. Eur J Anaesthesiol. 2012;29:208.

11. Dumonceau JM, Riphaus A, Aparicio JR, et al. Retraction of endorsement: European Society of Gastrointestinal Endoscopy, European Society of Gastroenterology and Endoscopy Nurses and Associates and the European Society of Anaesthesiology Guideline-non-anaesthesiologist administration of propofol for gastrointestinal endoscopy. Eur J Anaesthesiol. 2012;29: 208-209.

12. Hassan C, Rex DK, Cooper GS, et al. Endoscopist-directed propofol administration versus anesthesiologist assistance for colorectal cancer screening: a cost-effectiveness analysis. Endoscopy. 2012;44:456-464. 